\title{
Síndrome de estenose pilórica por adenocarcinoma de piloro
}

\section{Pyloric stenosis due to adenocarcinoma of the pylorus}

Célia Regina de Oliveira Garritano, tCBC-RJ'; José Carlos Guimarães Gomes, ACBC-RJ²; Rafael Domingos da Silvaż; Rafael Thome DA SILVA DE SOUSA ${ }^{3}$

\section{INTRODUÇÃO}

$\mathrm{O}$ $s$ adenocarcinomas, linfomas, sarcomas e tumores carcinoides representam de $1 \%$ a $5 \%$ de todos os tumores malignos gastrointestinais. O tipo mais comum de câncer de duodeno é o adenocarcinoma ${ }^{1,2}$, sendo responsável por $50 \%$ dos $\operatorname{casos}^{2,3}$. Foi descrito pela primeira vez por Hamberger, em 1746 ${ }^{3-5}$. Acomete mais o sexo masculino, sexta e sétima décadas de vida, ocorrendo, preferencialmente, na região periampular ${ }^{5}$.

O acometimento da primeira, terceira e quarta porções, é raro, especialmente na região pilórica, representando somente 0,35\% dos cânceres do sistema digestivo, com poucos relatos descritos na literatura ${ }^{1}$.

O reconhecimento precoce dos sintomas e a diferenciação com outras doenças de caráter benigno são importantes para que seja feita uma opção adequada de tratamento, reduzindo, assim, a morbidade e a mortalidade.

Relatamos uma síndrome de estenose pilórica ocasionada por adenocarcinoma de piloro.

\section{RELATO DO CASO}

Sexo masculino, 71 anos, com queixa de plenitude pós-prandial, desconforto em epigástrio, queimação retroesternal e dispepsia. Foi submetido a duas endoscopias digestivas altas (EDA), que não foram conclusivas. Fez tratamento com sintomáticos, obtendo melhora. Após quatro meses, os sintomas se agravaram, sendo realizada outra EDA, que evidenciou uma grande quantidade de resíduo gástrico, não sendo feita biópsia. Foi solicitada uma tomografia computadorizada (TC) abdominal, que evidenciou o estômago dilatado, cheio de resíduos, esvaziamento retardado, caracterizando um quadro obstrutivo (Figuras 1 e 2).

O paciente foi internado para realização de exames laboratoriais, correção de distúrbios hidroeletrolíticos e encaminhado para o tratamento cirúrgico. Durante a operação foi identificada dilatação gástrica e estenose cerrada do piloro, com aspecto sugestivo de malignidade.

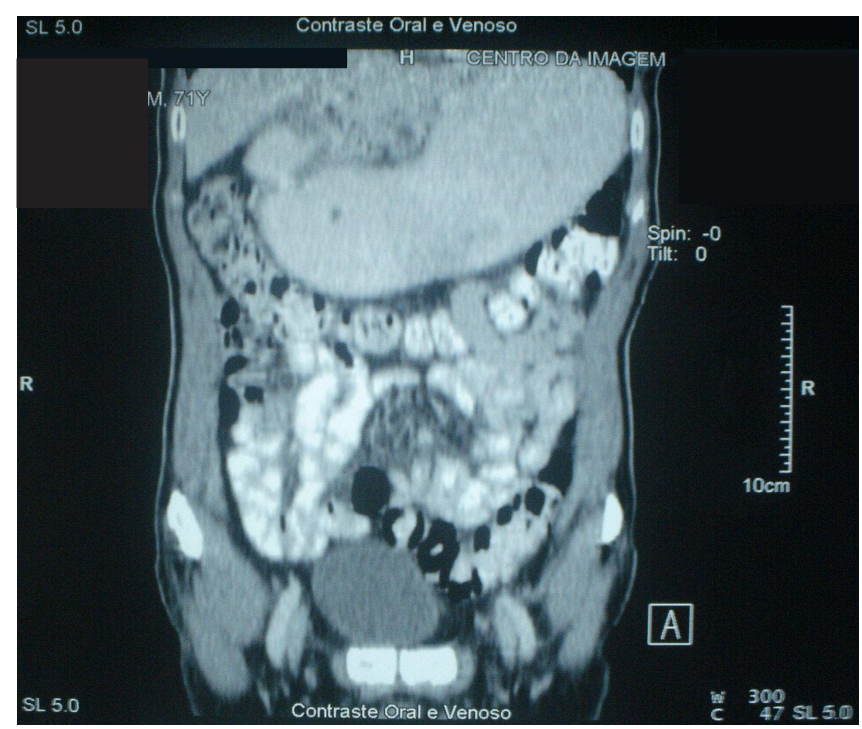

Figura 1 - TC abdominal - grande dilatação gástrica e estenose pilórica.

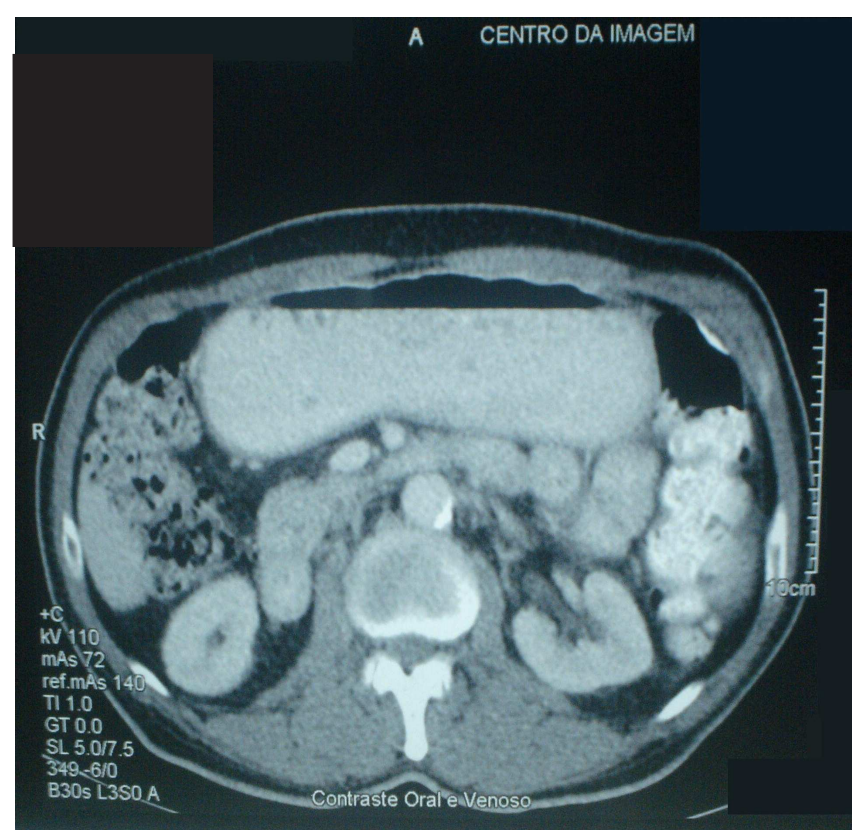

Figura 2 - TC abdominal - grande quantidade de resíduos no estômago.

Trabalho realizado no Hospital da Venerável Ordem Terceira de São Francisco da Penitência, Rio de Janeiro, RJ.

1. Professora Associada da Clínica Cirúrgica "A", da Universidade Federal do Estado do Rio de Janeiro, UNIRIO - Rio de Janeiro - RJ-BR; 2. Chefe da Cirurgia Geral do Hospital São Francisco da Penitência; 3. Graduação em Medicina da Universidade Federal do Estado do Rio de Janeiro, UNIRIO. 
Foi realizada a gastrectomia subtotal alargada. $\mathrm{O}$ laudo histopatológico da peça cirúrgica evidenciou adenocarcinoma de piloro, invadindo a serosa, com metástase para um linfonodo localizado na pequena curvatura. A evolução pós-operatória foi satisfatória, com o paciente obtendo alta hospitalar em boas condições clínicas.

\section{DISCUSSÃO}

A maioria dos casos de obstrução pilórica tem como principal suspeita clínica a doença ulcerosa péptica, e, pela raridade de adenocarcinomas nesta região, estes tumores não são comumente considerados como uma provável causa. A demora na identificação da doença é refletida na presença de metástases.

O diagnóstico é geralmente feito quando há complicações, como estenose, hemorragia, ou somente durante o ato cirúrgico. Isto acontece porque os sintomas inespecíficos ${ }^{1,3,5}$, como perda ponderal, náuseas, vômitos, anorexia, dor abdominal e anemia ferropriva, são também encontrados em outras doenças. Foi o que ocorreu no caso apresentado.

A endoscopia digestiva alta é o principal método para o diagnóstico, embora uma obstrução cerrada e a presença de resíduos no estômago possam dificultar o procedimento. No caso relatado, a EDA não foi conclusiva, talvez pelo fato de não ter sido realizado um preparo adequado para o completo esvaziamento gástrico com o intui- to de se ter uma boa visualização para coleta de material da lesão.

A tomografia computadorizada e a ressonância nuclear magnética são exames complementares à EDA, na tentativa de identificar a causa de uma obstrução pilórica, ou, se já estabelecido o diagnóstico de neoplasia, como métodos para o estadiamento. No caso apresentado, a TC evidenciou um quadro obstrutivo, sem identificar a causa.

O tratamento dos cânceres do intestino delgado é cirúrgico, sendo a duodenopancreatectomia o procedimento de escolha para os tumores da segunda porção do duodeno, podendo também ser utilizado para neoplasias de primeira e terceira porções ${ }^{4}$. No tumor de piloro, a indicação é a gastrectomia subtotal alargada, procedimento realizado no caso apresentado.

Os tratamentos quimio e radioterápico não influenciam a sobrevida dos pacientes ${ }^{4,5}$, não tendo sido também indicados no caso relatado.

A estatística dos poucos casos existentes indica uma taxa de sobrevida de cinco anos de somente $18 \%$ dos casos $^{4}$. O paciente encontrava-se em bom estado geral, sem evidência de metástase ou recidiva, após 20 meses do tratamento cirúrgico.

\section{Agradecimentos}

Os autores agradecem aos professores Miriam Orofino Gomes e Raul Barbosa de Sousa Junior pela revisão do texto em português e inglês, respectivamente.

\title{
A $B$ S S T R A C T
}

\begin{abstract}
We present a case of primary malignant pylorus neoplasia, emphasizing its rarity, the difficulty of diagnosis and the importance of an adequate prepare before the endoscopic procedure. Literature shows that tumors compromising the first, third and fourth segments of duodenum are rare, especially those of the pyloric area, representing only $0.35 \%$ of all malignant tumors of the gastrointestinal tract. Adenocarcinoma is the most common histological type, representing $50 \%$ of all malignant neoplasias at this site. The most common symptoms are weight loss, nausea, vomiting, abdominal pain, abdominal mass, sudden changes in intestinal habits and iron deficiency anemia secondary to chronic intestinal hemorrhage. Survival after five years is only 18\%. We report a case of a seventyone year-old male referring early satiety, epigastric pain, retrosternal burning and dyspepsia, with unspecific results in complementary exams, since complete gastric emptying was not achieved. As symptoms worsened, the patient was submitted to laparotomy, with identification of gastric dilation and severe pyloric stenosis, macroscopically suggesting malignancy. The chosen procedure was a subtotal gastrectomy. Adjuvant radiotherapy and chemotherapy were not used. The patient is been followed-up without tumor recurrence so far.
\end{abstract}

Key words: Neoplasms. Gastrointestinal neoplasms. Carcinoid tumor. Pyloric stenosis. Endoscopy, gastrointestinal.

\section{REFERÊNCIAS}

1. Navarrete S, Cantele H, Leyba JL, Vassallo M. Adenocarcinoma primario no ampullar de duodeno: reporte de caso y revisión de la literatura. Rev venez oncol. 2001;13(3):104-7.

2. Rangel MF, Silva MVM, Fernandes MJC, Ferreira MAS, Nóbrega LPS, Souza MG. Tumores malignos do intestino delgado. Rev Col Bras Cir. 2000;27(6):385-8.

3. Martins ACA, Martins Filho ED, Leão CS, Ferraz AAB, Ferraz EM Neoplasias do intestino delgado: experiência de cinco anos. Rev Col Bras Cir. 2001;28(5):342-6.
4. Siqueira PR, Nadal SR, Rodrigues FCM, Malheiros CA. Adenocarcinoma primário do duodeno. Rev Assoc Med Bras. 2002;48(3):242-4.

5. Nunes FC, Silva AL. Adenocarcinoma primário de duodeno. Rev Col Bras Cir. 2002;29(3):181-3.

Recebido em 02/06/2007

Aceito para publicação em 05/07/2007

Conflito de interesse: nenhum

Fonte de financiamento: nenhuma 


\section{Como citar este artigo:}

Garritano CRO, Gomes JCG, Silva RD, Sousa RTS. Tumor de piloro. Apresentação de caso. Rev Col Bras Cir. [periódico na Internet] 2013;40(5). Disponível em URL: http://www.scielo.br/rcbc
Endereço para correspondência:

Célia Regina de Oliveira Garritano

E-mail: cgarritano@oi.com.br 